\title{
A Low-Power Simplified-MEWS Scoring device for Patient Monitoring
}

\author{
Dirk de Jager $^{\dagger}$, Evangelos B Mazomenos ${ }^{\dagger}$, Anindo K Banerjee ${ }^{\ddagger}$, Koushik Maharatna ${ }^{\dagger}$ and Jeffrey S Reeve ${ }^{\dagger}$

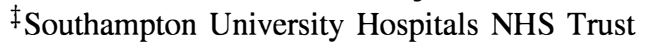 \\ ${ }^{\dagger}$ School of Electronics and Computer Science \\ University of Southampton \\ Southampton \\ Email: \{ddj07r, ebm07r, km3, jsr\}@ecs.soton.ac.uk
}

\begin{abstract}
The Modified Early Warning System is a paper based system used in general wards of hospitals to monitor patients health during the duration of the patient stay. Using this system, patient deterioration/improvement can rapidly be detected so as to assist and alert healthcare providers. In this paper we describe a simplified MEWS device which assists healthcare providers in assessing several of the patients vitals quantitatively, so as to allow the provider to focus on a qualitative assessment of the patient.
\end{abstract}

\section{INTRODUCTION}

Terminal conditions have been shown to be preceded by periods of abnormal physiological signals [1]. This has led to patients in hospital wards being monitored by nurses and clinicians using monitoring devices to observe individual vital signs and record abnormal signals. This patient data is logged onto a paper-based scoring sheet which is used to assess the patients' overall health. There are currently many different methods to assess the patients' overall health [2]-[5]. These so called "Track-and-Trigger" systems, provide a concise overview of the patient, as a patient score is calculated based on an algorithm which approximates when a patients condition has changed, giving a doctor a fast and clear representation of the patient's overall health. Paper based systems, although useful, have several commonly known downfalls [6] [7]. These are: data entry problems, illegibility, disorganisation, lack of privacy and inability to easily share information.

The Modified Early Warning Score (MEWS) is a popular patient scoring system for patients in wards, used in many of the United Kingdom National Health Service Trusts. The MEWS scoring system can be seen in Table I. From the table one can see how the scores are computed for a patient, where the patient score is continuously accumulated, so that a score which deviates from the norm of zero increases the patient score. When the score reaches four or above, the patient is required to be seen by a nurse in charge or higher level consultant. The score allows a patient carer to quickly assess whether a patient state has degraded and signal an alert that the patient requires further assistance, closer attention and that the patient is at risk of a critical event occurring. This is the reason the system is referred to as an early warning score, whereby a critical condition can be pre-emptively addressed.

The Southampton General Hospital in the United Kingdom

Permission to make digital or hard copies of all or part of this work for personal or classroom use is granted without fee provided that copies are not made or distributed for profit or commercial advantage and that copies bear this notice and the full citation on the first page. To copy otherwise, to republish, to post on servers or to redistribute to lists, requires prior specific permission and/or a fee. PERVASIVEHEALTH 2010, March 22-25, Munchen, Germany

Copyright @ 2010 ICST 978-963-9799-89-9

DOI 10.4108/ICST.PERVASIVEHEALTH2010.8870 use a MEWS scoring chart for monitoring patients in General, Intensive care, and High-dependancy wards. The information in this chart is entered manually in a vertical format thus allowing for a rapid horizontal assessment of the patients health in a graphical form.

The current MEWS system, which is optimised for paperbased entry, serves as an ideal example of why and how a Body Sensor Network (BSN) can support and assist healthcare providers within the hospital environment. We aim to show that it is feasible to use a BSN to assist patient carers, while increasing the time a carer can spend to qualitatively assess the patient, thereby improving the patient experience and assisting carers decisions in delivering high quality care.

\section{SySTEM OVERVIEW}

The MEWS System comprises of a single wireless sensor node per patient, which when attached to the patient, monitors several readings off of the patient and computes a score of the patients overall health as per the MEWS scoring table. By computing the score on the patient node, bandwidth requirements are decreased, and access to the patient data is available quickly and easily to the patient carer without external network dependency requirements. Using a low-power radio interface, allows confidential patient information to be geographically confined to within the ward environment thus decreasing the strict requirements of patient information while still ensuring confidentiality. Access to the information is communicated to the centralised hospital system via a base-station node as well as directly to the patient carer who using a PDA, smartphone or Laptop can view the patient data.

To realise a prototype of this system, a simplified form of the MEWS is used as shown in Table II. This simplified MEWS does not take into account Systolic Blood Pressure or Urine output, as appropriate low power unobtrusive sensors are not available for these measurements and is left for future work. For CNS Response, an accelerometer is used to gain a simplified understanding of whether the patient is alert or unresponsive. This is not a direct measurement of CNS response, but indirectly a good enough approximation.

An overview of the overall system working can be seen in Figure 1. 

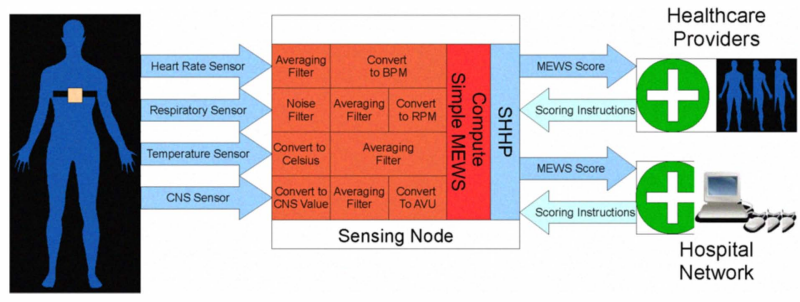

Fig. 1. MEWS Node

We have developed an application level protocol independent of the underlying wireless subsystem to ensure security and low-power usage of the communication channel. Once the data reaches the base-station, data can be changed to a hospital related communication protocol, such as HL7. The data communicated to the Patient Carers and base-station is called the Simple Helping Hand Protocol or SHHP.

\section{A. Simple Helping Hand Protocol}

Simple Helping Hand protocol (SHHP) is an application level protocol for body sensor networks to allow physiological parameters to be transmitted across a short-range wireless network. It ensures information is transmitted both securely and with the minimal amount of energy acceptable to the network. This allows for smaller battery, energy harvesting and energy constrained devices to be used, which in turn allow the patient more freedom of movement and the carer less invasive interaction performing tasks such as replacing of device batteries.

As SHHP is an application level protocol, it is compatible with many current WSN network level protocols. An overview of the generic packet structure can be seen in Table III. As SHHP has a data size of 49 - 105 bytes per packet, allows it to integrate into Industry Standard IEEE 802.15.4 Messages, TinyOS AM Messages [8] and Texas Instruments SimpliciTI messages [9], which are all protocols used in current Wireless Sensor Network Deployments.

TABLE III

Application Level Packet Structure

\begin{tabular}{|c|c|c|c|c|c|}
\hline Version & Priority & TTL & Message Type & Group & Data \\
\hline 4 bits & 4 bits & 4 bits & 4 bits & 1 byte & $49-105$ bytes \\
\hline
\end{tabular}

SHHP is a backward compatible protocol allowing for continuous development. To address concerns with development, it transmits 4 bits to the version of the protocol, thereby allowing sixteen concurrent revisions of the protocol. Four bits are assigned to prioritise packets destined across multihop networks, while the Time-to-Live field (TTL) ensures that messages are not continuously transmitted, and messages can be forwarded up to a maximum of fifteen times. The Message Type field indicates what type of message is being sent. Currently there are two message types: Data Messages and Instruction messages. Data messages are simple messages relaying information about a patient, while Instruction messages are messages which are used to redefine and reprogram the onnode scoring table and other modifiable system parameters. The next field, Group, indicates which group or patient ID the node is providing information about. This allows up to 255 patients to be concurrently monitored within the subnet of this system. The final field Data, is a dynamic sized field providing the information relative to the packet such as the individual MEWS Scores, Vital Sign Signals or Instructions with which to reprogram the node. Other fields such as destination, source, sequence number, CRC check and others are all gathered from the lower Network level of the OSI Stack, and are assumed to be known in this application level protocol, so therefore not required to be re-included at this level.

SHHP stores addresses of nodes interested in its data in its memory after having shared an encryption instruction with the sink nodes. A group is then defined for a patients data, allowing different nodes to communicate information about the same patient to and from different nodes and devices. This allows for a virtual connection to be established between the different nodes in the network.

Information stored in the Datafield of the generic packet descriptor, can be of two types, data and information packets. Data packets are packets which transport data about the node or the patient. It takes the form of key, value pairs with the first pair always a 32-bit date value describing the present date from midnight accurate to 20 micro-seconds. $20 \mu$ seconds has been chosen as it allows for a sampling frequency of $50 \mathrm{kHz}$, which is far higher than the highest required sampling frequency of intended physiological measurements, while still small enough to allow only a single 32-bit value to be sent on the start message transmission.

TABLE I

Modified Early Warning Score Parameter Table [4]

\begin{tabular}{|l||c|c|c|c|c|c|c|}
\hline Score & $\mathbf{3}$ & $\mathbf{2}$ & $\mathbf{1}$ & $\mathbf{0}$ & $\mathbf{1}$ & $\mathbf{2}$ & $\mathbf{3}$ \\
\hline Pulse & & $\leq 40$ & $41-50$ & $51-100$ & $101-110$ & $111-130$ & $\geq 131$ \\
\hline Respiratory Rate & $\leq 7$ & $8-10$ & & $11-14$ & $15-20$ & $21-29$ & $\geq 30$ \\
\hline Temperature & & $\leq 35.0$ & $35.1-36$ & $36.1-38$ & $38.1-38.5$ & $\geq 38.6$ & \\
\hline $\begin{array}{l}\text { CNS Response } \\
\text { or GCS* }\end{array}$ & & & agitation/confusion & $\underline{\text { Alert }}$ & $\underline{\text { Voice }}$ & $\underline{\text { Pain }}$ & $\underline{\text { Unresp }}$ \\
\hline Urine Output & & & 15 & 14 & $9-13$ & $\leq 8$ \\
\hline Systolic Blood Pressure & $\leq 10^{*}$ & $<0.5^{* *}$ & & $>0.5^{* *}$ & & & \\
\hline
\end{tabular}


1) Data: Table V defines how a data value is communicated in a Key-Value pair. The ID of the key describes precisely what information is defined in the following bits, while the size tells the node that the information is either 8-, 16-, 12-, 32-bits in length or 8-,12-, 16-,32-bit stream. The next key-value pair is located after the value, and therefore each packet is required to be read in order. If the information is a stream, no other keyvalue pair is included in the packet, while multiple different values can be sent if single values are used. If the information is not a stream, and multiple values are given for different sensors, a date value key-pair should be placed in between the data to define the time change between the initial packet time and the new time.

TABLE IV

Data Packet Key-Value Pair

\begin{tabular}{|c|c|l|}
\hline \multicolumn{2}{|c|}{ Key } & Value \\
\hline ID & Size & Number \\
\hline 4-bit & 4-bit & $\begin{array}{l}\text { 8-,12-,16-,32-bit values 8- } \\
\text {,12-,16-,32-bit stream }\end{array}$ \\
\hline
\end{tabular}

2) Instructions: Instruction packets are packets which are used to request data from the patient nodes, define patient details related to the patient which the node is monitoring, update the node's scoring table, or upgrade the software which is currently on the node. These packets are much more complex than normal data packets, and therefore require different descriptors for each instruction type.

3) Low Power: SHHP performs an assessment of the information before it is power expensively transmitted into the network. The valuation is based on the MEWS Score generated by the node, thus allowing more important higher MEWS scores to be transmitted before lower scores.

\section{HARDWARE}

The system is implemented using an RF2500T Wireless Sensor Node, which contains a MSP4302274 microcontroller, and CC2500 Wireless Transceiver. This device board along with a custom analog signal processing frontend.

\section{A. Sensors}

To obtain the temperature readings, a MAXIM 18BS20 digital temperature sensor was interfaced to the device. To obtain the respiratory rate, a simple custom developed capacitive sensor was developed to monitor changes due to muscle fluctuations around the lungs, while a heart rate was obtained via a hardware peak level detector circuit connected to a ECG circuit. The ECG data is then fed into an ADC input of the MCU.

The Central Nervous System (CNS) response is a measure of how conscious a patient is and is measured using either the Alert/Voice/Pain/Unresponsive (AVPU) scale [10] or the Glasgow Coma Scale (GCS) [11]. To approximate this scale using low-power sensors, A 3-axis accelerometer is used with a small light emitting diode (LED), a buzzer and a vibration motor connected to the MCU. The accelerometer senses patient movement, while the LED, buzzer and vibration motor are used as triggers to request the patient to respond. If the magnitude of movement is over a threshold value, the sensor records the patient as having responded to that input value. The sequence is initially started by blinking a small visible LED to the patient, if the threshold value is not reached by a movement such as tapping the device, the device sounds a brief chirp sequence from the buzzer, if there is no response to the buzzer, a vibration is started to try and detect a patient response. This allows the device to detect an approximate measure of CNS, while remaining reasonably unintrusive. As a response below the Alert state can be difficult to detect, the threshold value of the accelerometer is decreased at every stage to ensure that a very small response can be detected by the device.

TABLE V

ACCElERometer SENSOR USED FOR AVPU APPROXimation

\begin{tabular}{|l|c|c|}
\hline AVPU Scale & Trigger & MEWS Score \\
\hline Alert & LED & 0 \\
\hline Voice & Buzzer & 1 \\
\hline Pain & Vibrate & 2 \\
\hline Unresp & - & 3 \\
\hline
\end{tabular}

\section{B. Data-formatting and filtering}

Each sensor requires specialised processing to format the data into comprehensible signals relative to the MEWS Parameter Table. This requires each sensed reading to be filtered and converted to appropriate levels and signals. To realise this, several reusable libraries were written for the MSP430.

Noise filtering for the sensor is done using a software FIR filter of the form in equation 1 using $b$-value co-efficients taken from Matlab for filters of the required order. Due to memory constraints of the device, an 8th-order filter was used for the respiratory sensor, and 2nd order moving average windows were used for the Heart and Temperature readings.

TABLE II

A Simplified MEWS TABLE FOR MEWS SCORE APPROXIMATION

\begin{tabular}{|l||c|c|c|c|c|c|c|}
\hline Score & $\mathbf{3}$ & $\mathbf{2}$ & $\mathbf{1}$ & $\mathbf{0}$ & $\mathbf{1}$ & $\mathbf{2}$ & $\mathbf{3}$ \\
\hline Pulse & & $\leq 40$ & $41-50$ & $51-100$ & $101-110$ & $111-130$ & $\geq 131$ \\
\hline Respiratory Rate & $\leq 7$ & $8-10$ & & $11-14$ & $15-20$ & $21-29$ & $\geq 30$ \\
\hline Temperature & & $\leq 35.0$ & $35.1-36$ & $36.1-38$ & $38.1-38.5$ & $\geq 38.6$ & \\
\hline Alertness & & & & $\underline{\text { Alert }}$ & $\underline{\text { LED Blink }}$ & Vibrate & \\
\hline
\end{tabular}




$$
I_{v}(t)=\sum_{n=0}^{N} b_{i} x[n-i]
$$

Appropriate conversions are then performed on the data to obtain values relative to the MEWS scoring table. The new score is then accumulated with previous scores, and the result is then transmitted to the required healthcare providers and hospital systems subscribed to the patient data group, a connection supplied by SHHP.

\section{Evaluation}

As the device is not yet completed in hardware, and [12] has shown that battery requirements are normally the limiting factors in such systems, simulation was done to investigate how long the device would last with different batteries in different sampling conditions. Figure 2 shows that small lithium coin-cell batteries will allow the device to function for a sufficient period of time. For a six month hospital stay, the system can sample and forward patient information every 30seconds.

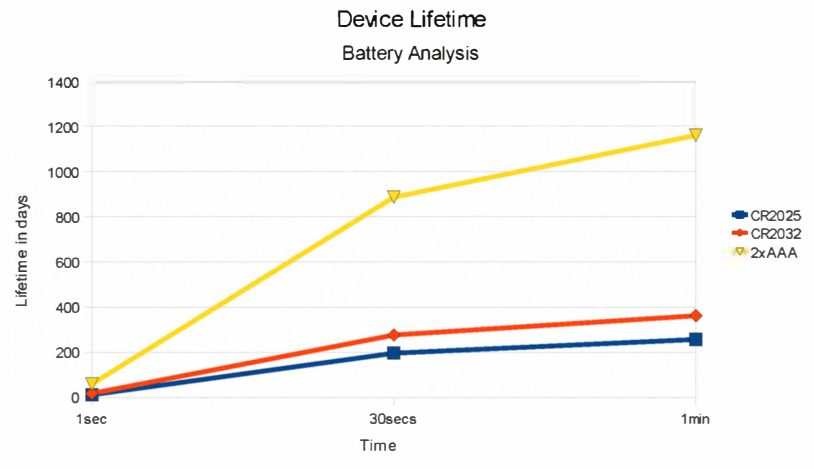

Fig. 2. Lifetime for sampling frequency

\section{CONCLUSIONS AND FUTURE WORK}

We have presented a simplified MEWS sensor device to assess a patients overall health and transmit the information back to interested parties. We propose a Wireless Sensor Network System to monitor patients unobtrusively using a novel lowpower application protocol aimed at physiological monitoring with small low power batteries. The device footprint which is smaller then current monitoring devices suggests that this form of device would be feasible to be used for long-term monitoring of patients.

This device computes a patient score independently of any external computing devices, and therefore suggests it can be used outside of the hospital environment. This in turn suggests that the device can be used as an enabler to preventative care of patients in risk groups. Patients can thus remain within their own home, while still being unobtrusively monitored and without the installation of an expensive pervasive environment. This will allow a home based carer or primary healthcare advisor visiting a patient to have an instant view of the patients health over a longer period of time without having directly observed the patient for the time period.

This device's use within the hospital is not intended as a replacement for other physiological measuring devices, but rather a decision support mechanism to ensure patient MEWS scores are accurately recorded, electronically stored, and easily accessible to healthcare providers.

Future work will include device evaluation in a hospital environment, compared against MEWS patient data. An assessment of whether the patient degradation is detected sooner or later than the paper based system will also be done. It is important to ensure high-level care of patients, so an assessment of the devices impact on both carers and patients will be performed to see whether the perceived care experience is improved or degraded.

\section{REFERENCES}

[1] R. Schein, N. Hazday, M. Pena, B. Ruben, and C. Sprung, "Clinical antecedents to in-hospital cardiopulmonary arrest," Chest, vol. 98, no. 6, pp. 1388-1392, Dec. 1990. [Online]. Available: http://www.chestjournal.org/cgi/content/abstract/98/6/1388

[2] F. Hourihan, G. Bishop, K. M. Hillman, K. Daffurn, and A. Lee, "The medical emergency team: a new strategy to identify and intervene in high-risk patients," Clinical Intensive Care, vol. 6, no. 6, pp. 269-272, 1995.

[3] D. R. Goldhill, L. Worthington, A. Mulcahy, M. Tarling, and A. Sumner, "The patient-at-risk team: identifying and managing seriously ill ward patients." Anaesthesia, vol. 54, no. 9, p. 853, 1999.

[4] C. Stenhouse, S. Coates, M. Tivey, P. Allsop, and T. Parker, "Prospective evaluation of a modified early warning score to aid earlier detection of patients developing critical illness on a general surgical ward," British Journal of Anaesthesia, vol. 84, no. 5, p. 663, May 2000. [Online]. Available: http://bja.oxfordjournals.org/cgi/content/abstract/84/5/663

[5] C. P. Subbe, R. Hibbs, E. Williams, P. Rutherford, and L. Gemmel, "ASSIST: a screening tool for critically ill patients on general medical wards," Intensive Care Med, vol. 28, no. S 1, p. S21, 2002.

[6] P. D. Clayton and G. Hripcsak, "Decision support in healthcare," International Journal of Bio-Medical Computing, vol. 39, no. 1, pp. 59-66, Apr. 1995. [Online]. Available: http://www.sciencedirect.com/science/ article/B7GH2-4C9KGFD-1D/2/7be60984598ccfec25664dd1 f47845d0

[7] J. Grimson, W. Grimson, and W. Hasselbring, "The SI challenge in health care," Commun. ACM, vol. 43, no. 6, pp. 48-55, 2000. [Online]. Available: http://portal.acm.org/citation.cfm?doid=336460.336474

[8] J. Hill, R. Szewczyk, A. Woo, S. Hollar, D. Culler, and K. Pister, "System architecture directions for networked sensors," SIGPLAN Not., vol. 35, no. 11, pp. 93-104, 2000. [Online]. Available: http://portal.acm.org/citation.cfm?id=356989.356998\&dl=GUIDE\& $\mathrm{dl}=\mathrm{ACM} \& \mathrm{idx}=356989 \&$ part $=$ periodical $\&$ WantType $=$ periodical $\&$ title $=$ ACM\%20SIGPLAN\%20Notices

[9] "SimpliciTI - RF software protocol." [Online]. Available: http: //www.ti.com/corp/docs/landing/simpliciTI/index.htm

[10] A. F. McNarry and D. R. Goldhill, "Simple bedside assessment of level of consciousness: comparison of two simple assessment scales with the glasgow coma scale*," Anaesthesia, vol. 59, no. 1, pp. 34-37, 2004. [Online]. Available: http://dx.doi.org/10.1111/j.1365-2044.2004.03526.x

[11] C. A. Kelly, A. Upex, and D. N. Bateman, "Comparison of consciousness level assessment in the poisoned patient using the alert/verbal/painful/unresponsive scale and the glasgow coma scale," Annals of emergency medicine, vol. 44, no. 2, p. 108113, 2004.

[12] D. Naydenova, J. Tomasik, D. Schroeder, and W. Krautschneider, "A portable SoC-Based ECG-System for $24 \mathrm{~h} \times 7 \mathrm{~d}$ operating time," IEEE Biomedical Circuits and Systems Conference, 2008, (in press). 